No significant differences in tremor measures were found when all welders were compared with all referents. Altogether twenty-five subjects had sCDT above the pathological level $1.7 \%$.

The subjects with high sCDT had increased tremor. Dominant hand: Tremor Intensity $0.21 \mathrm{~m} / \mathrm{s}^{2}$, compared to $0.15 \mathrm{~m} / \mathrm{s}^{2}$ (p < $0.001)$ for subjects with sCDT $<1.7 \%$. Non-dominant hand: Tremor Intensity $0.22 \mathrm{~m} / \mathrm{s}^{2}$ vs $0.15 \mathrm{~m} / \mathrm{s}^{2}$; $(\mathrm{p}<0.001)$.

The same pattern was found when the 16 welders with sCDT $\geq 1.7 \%$ were compared with welders with $\mathrm{sCDT}$ values $<1.7 \%$. The concentrations of the biological exposure indicators were similar in the welders with sCDT $\geq 1.7 \%$ compared to the other welders (B-Mn 12.8 vs $12.7 \mu \mathrm{g} / \mathrm{L}$; U-Mn 0.34 vs $0.36 \mu \mathrm{g} / \mathrm{g}$ cr.; S-Mn 1.3 vs $1.0 \mu \mathrm{g} / \mathrm{L})$.

Conclusions No effect of manganese exposure on tremor was observed, in contrast to a large effect from alcohol consumption. The results suggest that alcohol consumption can operate as a serious confounder in epidemiological studies of neurotoxicants.

\section{THORACIC SPINAL PAIN PREVALENCE IN THE MUSCULOSKELETAL DISORDERS SURVEILLANCE NETWORK OF THE FRENCH PAYS DE LA LOIRE REGION}

${ }^{1,2}{ }^{2}$ Natacha Fouquet, ${ }^{2}$ Julie Bodin, ${ }^{3}$ Alexis Descatha, ${ }^{2,4}$ Audrey Petit,
${ }^{2,5}$ Aline Ramond-Roquin, ${ }^{1}$ Catherine Ha, ${ }^{2,4}$ Yves Roquelaure. ${ }^{1}$ French Institute for Public
Health Surveillance, Department of Occupational Health, Saint-Maurice, France; ${ }^{2}$ LUNAM
University, University of Angers, Laboratory of Ergonomics and Epidemiology in
Occupational Health (LEEST), Angers, France; ${ }^{3}$ Inserm, Centre for Research in
Epidemiology and Population Health (CESP), U1018, "Population-Based Epidemiological
Cohorts" Research Platform, Villejuif, France; ${ }^{4}$ CHU Angers, Angers, France; ${ }^{5}$ LUNAM
University, University of Angers, Department of General Practice, Angers, France

10.1136/oemed-2014-102362.74

Objectives Prevalence studies of thoracic spinal pain (TSP) in the working population are scarce. The epidemiological surveillance of musculoskeletal disorders (MSDs), implemented in 2002 by the French Institute for Public Health Surveillance, allows the study of the prevalence of TSP in a large sample of workers. The aim of this study is to present the prevalence of TSP during the preceding 7 days in the Pays de la Loire region's workforce according to age, combination with low back pain and neck pain, occupational category and industry sector, separately in men and women.

Method A random sample of 3710 workers (58\% of men) aged 20-59 years, representative of the regional workforce, was constituted between 2002 and 2005. Medical and occupational data were gathered by questionnaire.

Results The prevalence of TSP was higher among women (17.4\%) than men (9.2\%), without age difference. Only $15.2 \%$ of TSP in men and $15.7 \%$ in women was declared without low back pain or/and neck pain. Among men, lower-grade whitecollar workers were more likely to report TSP (16.6\%) than other occupational categories workers (upper white-collar and professionals: $7.2 \%$, technicians/associate professionals: $6.5 \%$, blue-collar workers: 9.7\%). Among women, upper white-collar and professionals were more likely to report TSP $(25.6 \%)$ than the others (technicians/associate professionals: 17.0\%, lowergrade white-collar workers: $17.1 \%$, blue-collar workers: 16.7\%). The study did not suggest a significant difference in the prevalence of TSP according to sectors in either men or women.

Conclusions This study shows that, among workers, TSP is frequent and often combined with low back pain or neck pain.

\section{PREDICTION OF MENTAL OUTCOMES BY EARLY CONDITIONS AFTER OCCUPATIONAL INJURIES}

1,2 Leon Guo, ${ }^{3}$ Kuan-Han Lin, ${ }^{4}$ Nai-Wen Guo, J Judith Shu-Chu Shiao, ${ }^{6}$ Shih-Cheng Liao, ${ }^{7}$ Pei-Yi Hu, ${ }^{7}$ Jin-Huei Hsu. ${ }^{1}$ Environmental and Occupational Medicine, National Taiwan University College of Medicine (NTU) and NTU Hospital, Taipei, Taiwan; ${ }^{2}$ Institute of Occupational Medicine and Industrial Hygiene, National Taiwan University School of Public Health, Taipei, Taiwan: ${ }^{3}$ Department of Social Medicine, College of Medicine, National Taiwan University, Taipei, Taiwan; ${ }^{4}$ Institute of Behavioral Medicine, National Cheng Kung University, Tainan, Taiwan; ${ }^{5}$ Department of Nursing, College of Medicine, National Taiwan University (NTU) and NTU Hospital, Taipei, Taiwan; ${ }^{6}$ Department of Psychiatry, National Taiwan University Hospital, Taipei, Taiwan; 7 Institute of Occupational Safety and Health, Councils of Labor Affairs, Taipei, Taiwan

\subsection{6/oemed-2014-102362.75}

Objectives Psychiatric diseases have been an important complication after occupational injuries. This study aimed to determine early factors predicting psychological health outcomes at twelve months after occupational injuries.

Method The study candidates were workers who sustained occupational injuries and were hospitalised for 3 days or longer in 2009. A self-reported questionnaire was sent to them at three months after injury. The questionnaire inquired about demographics, severity of injury, working status, personal factors, as well as included a psychometric instrument Brief Symptom Rating Scale (BSRS-50). At one year, all participants were invited again to complete a questionnaire including BSRS-50.

Results A total of 853 workers completed the questionnaire at three months and 12 months after injury. Among them, 84 (9.3\%) had general severity index (GSI) of 70 or higher at 12 months after injury, indicating poor psychological condition. Using multivariate logistic regression analysis for mutual adjustment, predictive factors for elevated GSI were found to include dismemberment or affected physical appearance (OR 1.7, 95\% CI 1.0-3.1), BSRS at 3 months after injury (OR 8.8, 95\% CI 5.4-14.4), and having to leave original workplace (OR 2.5, 95\% CI 1.4-4.3). The findings indicated that severity of injury, early psychological condition, and workplace accommodation to allow returning to original workplace are important factors for later psychological health after occupational injury.

Conclusions Among workers sustaining occupational injuries, psychological condition could have been affected at 12 months after injury. Predictors of the psychological condition were identified, to allow for possibility of early intervention.

\section{CIRCULATORY SYSTEM DISEASE MORTALITY AMONG FRENCH URANIUM MINERS}

${ }^{1}$ Damien Drubay, ${ }^{1}$ Sylvaine Caër-Lorho, ${ }^{2}$ Pierre Laroche, ${ }^{2}$ Alain Acker, ${ }^{1}$ Dominique Laurier, ${ }^{1}$ Estelle Rage. ${ }^{1}$ Institute for Radiological Protection and Nuclear Safety, Fontenay Aux Roses, France; ${ }^{2}$ AREVA, Paris, France

\subsection{6/oemed-2014-102362.76}

Objectives There is currently no consensus for an association between risk of Circulatory System Diseases (CSD) and low exposure to ionising radiation. The aim is to study the relationship between CSD mortality and radon exposure in the French cohort of uranium miners considering classical CSD risk factors.

Method The French cohort includes 5086 uranium miners followed from 1946 through 2007. Among the 1935 deaths, 442 were from CSD, including 167 cases of IHD and 105 cases of CVD. Annual radon exposures were assessed individually. A 
nested case-control study was performed to collect individual information concerning classical CSD risk factors (weight, height, smoking status, blood pressure, blood glucose and cholesterolemia) from medical files for 76 cases of death from CSD (including 26 from IHD and 16 from CVD) and 237 controls, matched for attained age and birth date and counter-matched for cumulative radon exposure.

Results In the whole cohort, a significant association with cumulative radon exposure was observed for CVD mortality, but not for CSD or IHD mortality. In the case-control study, no significant unadjusted Odd-Ratio for cumulative radon exposure was observed for any endpoint. Analyses adjusted on CSD risk factors, for which missing data do not exceed 25\%, are ongoing.

Conclusions The issue of CSD associated to ionising radiation is crucial for radiation protection. The present study, allowing to consider individual data on major classical CSD risk factors, will contribute to improve knowledge on the effects of low dose exposure.

\section{CARBON NANOTUBE EXPOSURE ASSESSMENT FOR A STUDY ON EARLY BIOLOGICAL EFFECTS; THE CANTES STUDY}

${ }^{1}$ Eelco Kuijpers, ${ }^{2}$ Roel Vermeulen, ${ }^{1}$ Peter Tromp, 'Wouter Fransman, ${ }^{1}$ Derk Brouwer, ${ }^{3,4}$ Lode Godderis, ${ }^{2}$ Jelle Vlaanderen, ${ }^{1,2}$ Cindy Bekker, 'Anjoeka Pronk. ${ }^{1} T N O$, Zeist, The Netherlands; '2IRAS, Utrecht University, Utrecht, The Netherlands; ' ${ }^{3}$ Occupational, Environmental and Insurance Medicine, KU Leuven, Leuven, Belgium; ${ }^{4}$ External Service for Prevention and Protection at Work, Idewe, Heverlee, Belgium

10.1136/oemed-2014-102362.77

Objectives To assess personal exposure to carbon nanotubes for a study of early effect biomarkers among workers exposed to carbon nanotubes (CNTs).

Method Three major job categories were identified in the exposed factory: production, R\&D and office. For qualitative assessment personal 8-hr-TWA inhalable dust samples $(\mathrm{n}=5)$ were collected in all job categories and analysed by SEM-EDX. For quantitative assessment 8-hr TWA samples $(\mathrm{n}=30)$ were collected from the production and R\&D workers and analysed for elemental carbon, corrected for soot using SEM/EDX. Job activities were recorded during all measurements.

Results The qualitative analyses demonstrated the presence of CNTs in the personal breathing zone of production, R\&D and office workers. CNT agglomerates with particle sizes between $500 \mathrm{~nm}$ and $100 \mu \mathrm{m}$ were identified for production and $R \& D$ workers and between $500 \mathrm{~nm}$ and $5 \mu \mathrm{m}$ for office workers. No single CNTs were identified. The quantitative analyses demonstrated geometric mean (GM) inhalable CNT levels of $42.6 \mu \mathrm{g} / \mathrm{m}^{3}$ (min-max: 1.4-1186.5) and $4.6 \mu \mathrm{g} / \mathrm{m}^{3}$ (min-max: 0.2-59.5) for the production and R\&D workers, respectively.

Conclusions We identified exposure to clusters of CNT in production, R\&D and office workers of the CNT production facility. As expected GM exposure was higher for production than for R\&D workers, however considerable variability was observed. Additional quantitative measurements in office and production workers are ongoing. For the full set, analyses of within- and between-worker variability and activity as a determinant will be performed. This information will be used to assess personal exposure in a cross-sectional study of early health effects in the CANTES study.

\section{LUNG CANCER RISK AMONG MINNESOTA TACONITE MINING WORKERS}

Elizabeth Allen, Bruce Alexander, Jeffrey Mandel, Richard Maclehose, Gurumurthy Ramachandran. University of Minnesota, Minneapolis, MN, USA

\subsection{6/oemed-2014-102362.78}

Objectives The mining and processing of taconite results in exposures to non-asbestiform amphibole and non-amphibole minerals. Previous studies have shown that taconite mining workers are at an increased risk for developing lung cancer and mesothelioma and duration of employment has been shown to contribute to the risk of mesothelioma incidence. The objective of this analysis is to examine the relationship between duration of employment and lung cancer among Minnesota taconite workers.

Method Among a cohort of 44243 taconite workers, 1721 cases of lung cancer were identified and matched by five-year age interval to two controls. Total duration of employment was abstracted from individual work records.

Results Among the 5159 workers included in the analysis, 55\% worked less than one year and 15\% worked $1-5$ years, $5-15$ years and more than 15 years. The mean duration of employment among cases and controls was 6.7 and 7.2 years respectively. A conditional logistic regression analysis did not show an increased risk for development of lung cancer among those who worked $1-5$ years $(\mathrm{OR}=$ 1.2, 95\% CI: $0.99,1.38), 5-15$ years $(\mathrm{OR}=0.94,95 \%$ CI: 0.79 , $1.11)$, and more than 15 years $(\mathrm{OR}=0.90,95 \% \mathrm{CI}: 0.75,1.08)$ as compared to those who worked less than one year.

Conclusions Risk for development of lung cancer does not appear to be associated with duration of employment in the taconite industry. Future analyses will explore specific exposures to airborne particulates, including silica and non-asbestiform amphiboles, in this population.

\section{USING MACHINE LEARNING TO EFFICIENTLY USE MULTIPLE EXPERTS TO ASSIGN OCCUPATIONAL LEAD EXPOSURE ESTIMATES IN A CASE-CONTROL STUDY}

${ }^{1}$ Melissa C Friesen, ${ }^{1}$ Sarah I Locke, ${ }^{2}$ Dennis Zaebst, ${ }^{2}$ Susan Viet, ${ }^{3}$ Susan Shortreed, 'Yu-Cheng Chen, ${ }^{4}$ Dong-Hee Koh, ${ }^{5}$ Larissa Pardo, ${ }^{6}$ Kendra L Schwartz, ${ }^{7}$ Faith G Davis, ${ }^{8}$ Patricia A Stewart, ' ${ }^{1}$ Joanne S Colt, ${ }^{9}$ Mark P Purdue. ${ }^{1}$ Occupational and Environmental Epidemiology Branch, NCI, Bethesda, MD, USA; ${ }^{2}$ Westat, Rockville, MD, USA; ${ }^{3}$ Biostatistics Unit, Group Health Research Institute, Seattle, WA, USA; ${ }^{4}$ Carcinogenic Hazard Branch, National Cancer Center, Seoul, Republic of Korea; ${ }^{5}$ Emory University Rollins School of Public Health, Atlanta, GA, USA; ${ }^{6}$ Department of Family Medicine and Public Health Sciences and Barbara Ann Karmanos Institute, Wayne State University School of Medicine, Detroit, MI, USA; ' $S$ School of Public Health, University of Alberta, Edmonton, AB, Canada; ${ }^{8}$ Stewart Exposure Assessments, LLC, Arlington, VA, USA

\subsection{6/oemed-2014-102362.79}

Objectives We applied machine learning approaches to efficiently assist multiple experts to transparently estimate occupational lead exposure in a case-control study of renal cell carcinoma.

Method We used hierarchical cluster models to classify the 7154 study jobs with occupational history and job/industry questionnaires into 360 groups with similar responses. Each group was reviewed independently by two or three experts and was assigned probabilities of lead exposure $(<5 \%, \geq 5-<50 \%$, $\geq 50 \%)$ for three time periods $(<1980,1980-1994$, $\geq 1995)$. When the group's mean response pattern suggested within-group exposure variability, experts identified programmable conditions that defined the rating differences where possible or flagged the group for further review. After splitting jobs that overlapped 